\title{
Research on the factors affecting the accuracy of three-dimensional reconstruction model of Rotor UAV archaeological sites
}

\author{
Xin Zheng ${ }^{1,2,3}$, Yungang $\mathrm{Hu}^{1,2,3}$ \\ ${ }^{1}$ Beijing Advanced Innovation Center for Future Urban Design, Beijing University of Civil Engineering and Architecture, Beijing \\ 100044, China; hyg@bucea.edu.cn (Y.H.); 210813J117012@stu.bucea.edu.cn (X.Z.) \\ ${ }^{2}$ School of Geomatics and Urban Spatial Informatics, Beijing University of Civil Engineering and Architecture, Beijing 100044, \\ ${ }^{3}$ Beijing Key Laboratory for Architectural Heritage Fine Reconstruction \& Health Monitoring, Beijing 100044, China.
}

Commission IV, ICWG IV/III, WG IV/4

KEY WORDS: oblique photography; modeling accuracy; cultural heritage; archaeological site

\begin{abstract}
:
The work of cultural heritage protection has risen to the national strategic level in China in recent years. More and more high and new technologies are applied in the fields of cultural relics and archaeology, heritage protection, etc., including the drone photography technology. Because the cultural protection industry has high requirements for the accuracy of reconstruction results, it puts forward higher requirements for the three-dimensional reconstruction of drones. In this paper, the factors affecting the reconstruction accuracy of the rotor unmanned opportunity are analyzed and summarized in the archaeological protection of cultural relics, and the technical integration of the oblique photography technology in archaeological work is carried out to improve the quality of modeling results.
\end{abstract}

\section{INTRODUCTION}

It is mentioned in 11.4 of the SDGs that cultural heritage is a common civilization wealth of all mankind and needs to strengthen efforts to protect and safeguard the world's cultural and natural heritage. But more and more ruins are facing devastating destruction and demise as time goes by and the environment changes. The preservation of heritage and archaeological sustainability has become a major strategic need for the country to enhance cultural self-confidence. The preservation of a large number of ruins and the perpetuation of digital information will certainly be put on the agenda.

The digital protection of the ruins begins with a threedimensional reconstruction of the ruins and a more elaborate reconstruction model. Then through the virtual restoration, virtual restoration, and three-dimensional real-life display of the reconstruction model, not only can the digital records of each period of archaeology be recorded, but also reliable support for archaeological excavation and subsequent scientific protection. UAV tilt photography is an efficient 3D reconstruction technology developed in recent years. It is characterized by low cost, easy operation, high flexibility, and is suitable for a wide range of $3 \mathrm{D}$ reconstruction work.

The archaeological work of the ruin has high requirements for the accuracy of $3 \mathrm{D}$ reconstruction. It is not only necessary to ensure the integrity of the model, but also has a good mapping effect. It also has higher requirements on the resolution of the model, and sometimes needs to achieve millimeter precision. UAVs will be affected by many factors in actual work, such as the selection of drones and lenses, the layout of field survey parameters and control points, and the selection of software and hardware for later data processing. Through reading relevant research results, most scholars have studied one of them, and did not conduct an overall analysis of the influencing factors. Due to the large number of influencing factors, the research results are too much, and the lack of collation and summary makes it difficult to obtain the required information directly and efficiently.

Therefore, this paper attempts to analyze the high-precision requirements of the tilting photography of the drones of archaeological sites from the aspects of the UAV platform equipment, aerial camera parameters and route parameter settings, ground control point layout and data processing hardware and software. The method and the method of summarizing the research results are used to comprehensively analyze the factors affecting the reconstruction model and determine the factors that mainly affect the modeling accuracy. At the same time, according to the special work requirements of the actual site archaeology, improve the working methods to improve the quality of the reconstruction results, in order to meet the relevant needs of archaeological experts.

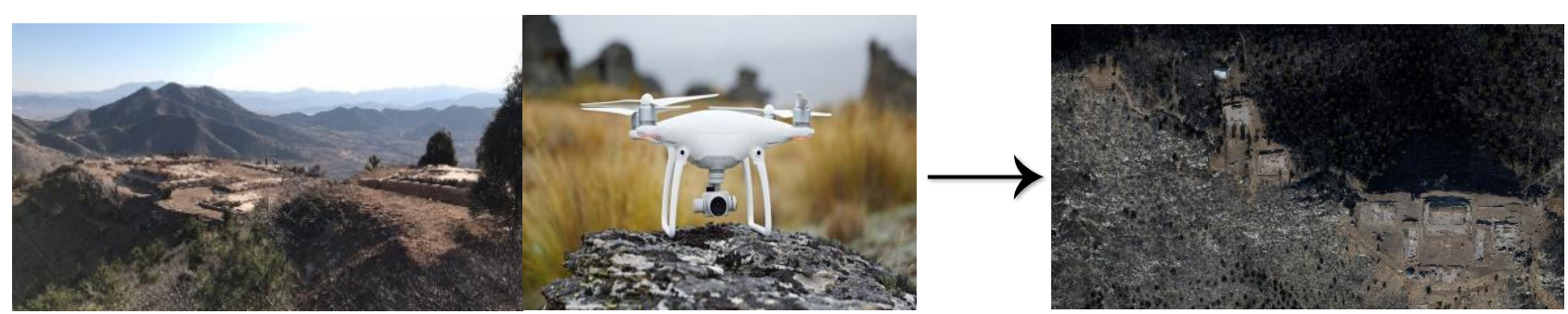

Figure 1. Unmanned Aerial Vehicle Used in Archaeology of Relics 


\section{INFLUENCING FACTORS OF RECONSTRUCTION ACCURACY OF ROTOR UAV}

\subsection{Technical route}

In order to determine the degree of influence of different factors on the modeling results, this paper uses different research methods to analyze and summarize the three main aspects of the selection of the UAV equipment, the related settings of the field survey and the data processing. When the UAV is conducting an aerial survey task, many sensors carried by itself will generate a large number of parameters and direct image data quality. The method of finding the most frequently occurring parameters from the calculation formula of the relevant derivative parameters is Formula analysis method. In the selection of UAV equipment, the research method based on formula analysis will be combined with the latest research results. In the two aspects of aerial survey and post-processing, the method of summarizing and sorting is mainly adopted, and the method of formula analysis will be taken into consideration when specific factors are involved. The specific technical route is shown in Figure 2.

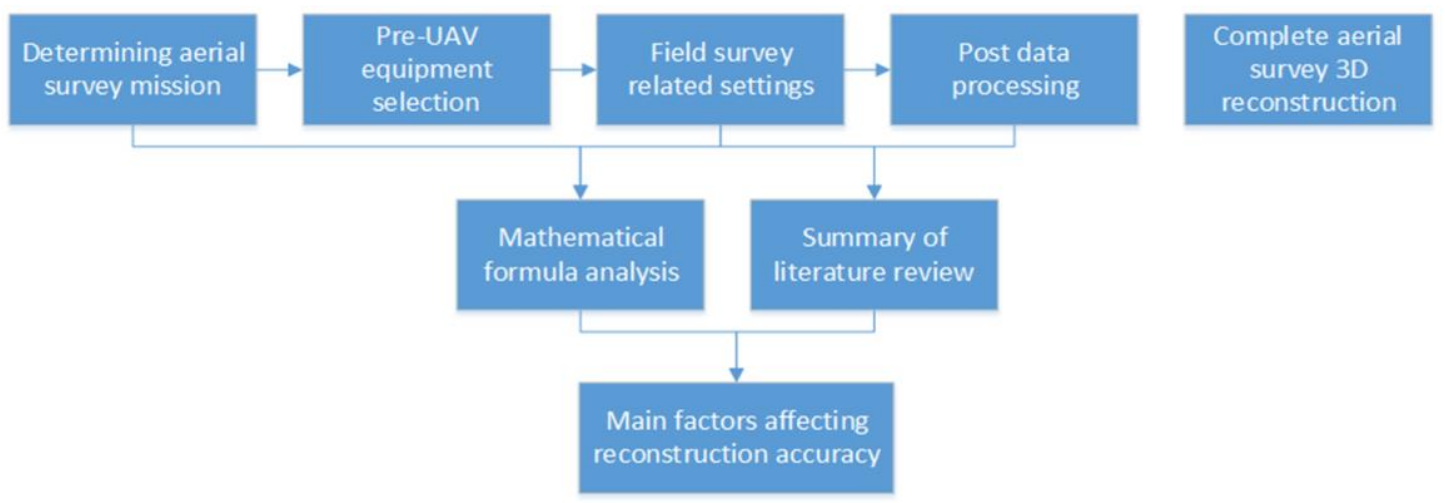

Figure 2. Analysis Chart of Main Influencing Factors on Reconstruction Accuracy

\subsection{UAV selection and parameter settings}

Some scholars have proved through research that the UAV tilt photography technology can also play a big role in the field of architectural heritage (Li, 2018), in the wild ruins (Sun et al., 2018), ancient tower, ancient buildings (Sun et al., 2017) and grottoes. The three-dimensional reconstruction of cultural heritage archaeology ( $\mathrm{Li}, 2019)$ has an important position. These scholars' articles have carried out an accuracy analysis on the reconstruction results, and the accuracy of the UAV tilt photography can meet the needs of the industry.However, the question of how the drone used is chosen is not described and explained. Choosing the right drone is crucial before archaeological work. It is important to consider the choice of drone platform and lens. The choice of camera and lens is a more important aspect.

\subsubsection{Selection of Unmanned Aerial Vehicle Platform: This} problem will be analyzed and summarized from the following seven aspects: Bearing capacity, Stability, Reliability, Endurance, Cost, Volume and Flexibility. By reading and summarizing the relevant literature, the following table can be obtained:

\begin{tabular}{|l|l|l|l|}
\hline & $\begin{array}{l}\text { Four- } \\
\text { rotor }\end{array}$ & $\begin{array}{l}\text { Six- } \\
\text { rotor }\end{array}$ & $\begin{array}{l}\text { Eight- } \\
\text { rotor }\end{array}$ \\
\hline Bearing capacity & $\star$ & $\star \star$ & $\star \star \star$ \\
Stability & $\star$ & $\star \star$ & $\star \star \star$ \\
Reliability & $\star$ & $\star \star$ & $\star \star \star$ \\
Endurance & $\star$ & $\star \star$ & $\star \star \star$ \\
Cost & $\star \star \star$ & $\star \star$ & $\star$ \\
Volume & $\star$ & $\star \star$ & $\star \star \star$ \\
Flexibility & $\star \star \star$ & $\star \star$ & $\star$ \\
\hline
\end{tabular}

Table 1 Comparison of related factors of multi-rotor UAV

According to the needs and environment of different tasks and the selected UAVs in the above table, it is an important prerequisite for obtaining a high-precision model to select the appropriate UAV type for different aerial survey tasks under the condition of cost and equipment conditions.

2.2.2 Selection of camera and lens: Selecting a drone camera mainly considers the number of camera lenses, sensor size, 
camera pixels, camera focal length, and resolution (Wu et al., 2016). Resolution factors (including image resolution, ground resolution, and model resolution) need to be considered.

The ground resolution (spatial resolution) represents the actual ground distance represented by a pixel in the pixel. The mapping scale can be combined with the ground resolution by equation, that is " 1 foot $=96$ pixels $=0.0254 \mathrm{~m}$, so that 1 pixel $=2.65 \times 10^{-}$ 4" $\mathrm{M}$ can be obtained. The general conversion relationship between scale and them is as follows: Scale $=$ distance on the graph / actual distance $=1:\left(\right.$ resolution $\left./ 2.65 \times 10^{4}\right)$

For example, a tilt image with a resolution of $5 \mathrm{~cm}$ can be easily calculated to have a scale of $1: 180$, which means that it can meet the requirements of a 1:200 large scale topographic map. In terms of model accuracy, it is necessary to compare the checkpoint coordinates in the 3D model with the actual GPS measured coordinates of the checkpoint for accuracy assessment. This is the most accurate way to calculate the three-dimensional reconstruction model of oblique photography. In the industry, there is a saying that the model accuracy is 3 times the ground resolution, that is, the model elevation accuracy of tilt photography is generally three times that of the ground resolution (GSD).

The number of lenses is mainly reflected in the work efficiency of the UAV tilt photography work, and the impact on the accuracy is mainly reflected in the distortion of the camera. Professional tilt camera components are typically five lenses, but there are also dual, triple and four lens cameras. Most of these lenses are measuring camera lenses, the lens is less distorted, and the image quality obtained is also higher. the lens is less distorted, and the quality of the impact is higher. Most of the cameras selected for single-lens drones are non-measurement cameras, and the DJI Phantom series of consumer drones are the most representative. Many scholars have carried out experiments on the working ability of consumer-grade UAVs in the field of 3D reconstruction. The experimental results show that after better parameter setting and distortion correction, it is also applicable to consumer-grade UAVs to achieve the industry standard for aerial survey 3D reconstruction (Liu, 2016).

Although the five-lens camera is a professional device for tilt photography, there is almost no distortion. However, the use of a five-lens drone also requires consideration of the focal length of the down-camera and tilt-camera. The focal length of the camera is long, and the smaller the field of view, the more image texture can be obtained; on the contrary, the focal length is shorter, the angle of view is large, and the image distortion is more serious. In general, the GSD of the lower camera should be equivalent to the GSD mid of the side view camera (Yang et al., 2016):

$$
\begin{gathered}
\mathrm{GSD}=\frac{\delta \times \hbar}{f_{\text {down }}}=G S D_{\text {mid }}=\frac{\delta \times h}{f_{\text {side }} \times \cos \alpha} \\
f_{\text {side }}=\frac{\delta \times \hbar}{\cos \alpha}
\end{gathered}
$$

In the formula , $\alpha$ is the camera tilt angle. When the tilt angle is set to $45^{\circ}$, the focal length of the side view camera is generally 1.4 times the focal length of the down view camera. Therefore, when tilting photography, the focal length of the side view camera is generally longer than that of the lower view camera. Choosing the right lens for matching can effectively control image distortion and improve $3 \mathrm{D}$ reconstruction accuracy.

2.2.3 Distortion of the camera lens: In recent years, with the rise of consumer-grade drones, there have been many studies on its applicability and mapping (Zhang et al., 2018). The nonmeasurement camera mounted on the consumer drone has its own distortion, which will seriously affect the accuracy of the acquired photos, resulting in the error of the three-point accuracy of the air directly affecting the 3D reconstruction result (Liao et al., 2018). The distortion error formula is as follows:

$$
\begin{aligned}
& \Delta \mathrm{x}=\mathrm{x} \times\left(k_{1} \times \gamma^{2}+k_{2} \times \gamma^{4}+k_{3} \times \gamma^{6}\right) \\
& \Delta \mathrm{y}=\mathrm{y} \times\left(k_{1} \times \gamma^{2}+k_{2} \times \gamma^{4}+k_{3} \times \gamma^{6}\right)
\end{aligned}
$$

Where $\mathrm{k}_{\mathrm{i}}$ is the radial distortion coefficient of the objective system, $\gamma$ is the radial of the image point, and the radial approximation formula. The camera distortion error is the error caused by the nature of the camera itself, which is an accidental error, but its effect is systematic. Correction must be performed before 3D reconstruction (Zheng., 2015). It is generally corrected by establishing the test field method (Yang., 2009). In addition to the test field method, there is also a method of camera calibration. The commonly used camera calibration method is

"Zhang Zhengyou” (checkerboard) camera calibration method The accuracy of $3 \mathrm{D}$ reconstruction of sequence images after camera distortion correction is greatly improved, so camera lens 
distortion correction is very important and important in controlling the accuracy of $3 \mathrm{D}$ reconstruction.

\subsection{Route parameters and layout of image control points}

2.3.1 Route parameter setting: The route setting of the drone is the most critical for the tilt photography work. Many problems need to be considered when setting the route parameters, including, for example, flight speed, image overlap, altitude (Liao et al., 2011). Through research and summary, it is found that the route height (including absolute altitude, relative altitude and average altitude) is more important. In order to achieve the image ground resolution of the project, it is necessary to set the elevation of the route for the terrain of the survey area.

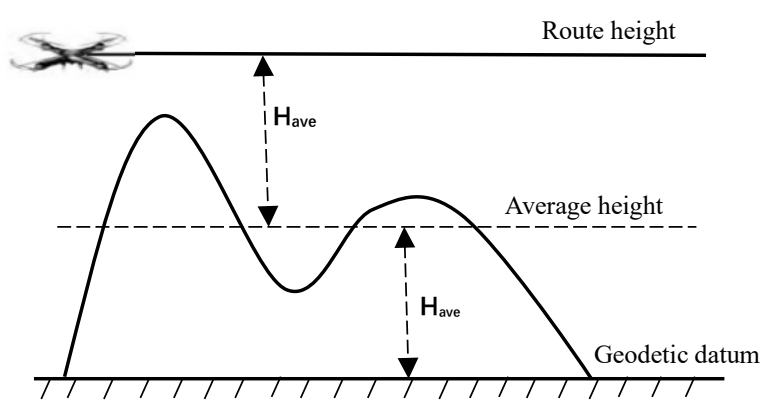

Figure 3 Schematic diagram of the average height of the relief terrain

When the terrain of the survey area is relatively flat, it can be approximated that the average altitude is the actual altitude. However, in the environment where the terrain of the survey area is relatively complicated and the terrain is very undulating, it is necessary to carefully set the altitude. If the average elevation is selected as the calculation standard for the camera resolution, the ground resolution of the image at the lowest point of the terrain will be much smaller than the set standard. However, in order to meet the ground resolution of the lowest point, it may cause the route to be too low, and even the drone may be in danger of hitting the mountain. Therefore, it is necessary to calculate and adjust the minimum ground resolution of the average altitude and the minimum point until the overall demand of the aerial survey project is met.

In addition to the aeronautical altitude, the setting of the overlap has a great influence on the accuracy of the three-dimensional reconstruction. In the relevant regulations, when the UAV is used for tilt photography, the heading overlap rate should be $60 \%$ to $80 \%$, and the side overlap rate should be no less than $70 \%$.
However, in practical applications, the set overlap rate tends to deviate from the actual overlap rate of the final captured photos. Especially when the local shape is large, this requires local design parameters according to terrain and ground resolution requirements (Jin et al., 2016).

2.3.2 Control points setting: In view of the general protection of cultural heritage, low-altitude drone aerial survey missions are generally completed in smaller survey areas, and control point layout should take this into account. In the actual UAV aerial survey work, there are many unreasonable control points. The control point layout and density have a great influence on the quality of UAV image products. The traditional photogrammetry method is adopted, and the number of control points is large, resulting in a large number of control points. Field work will become difficult. Therefore, low-altitude small drone aerial photography should not adopt the traditional aerial survey control point layout (Mai et al., 2012). The aerial photography of low-altitude small unmanned aerial vehicles can be divided into two categories according to the layout of control points: (1) GNSS assisted aerial survey of unmanned aerial vehicles without control point data; (2) aerial photography of drones with uniform control points, mainly used for reference The control point layout method of traditional aerial photography. At present, the aerial photography operation of the UAV mainly adopts the second method. The control points are three-dimensionally and evenly distributed in the measurement area, and the control point layout scheme of the edge of the measurement area is enhanced with high precision, and the digital mapping in the large area is 1:2000. On the issue of the control point layout scheme, many scholars have carried out research and proposed a solution. It is considered that the method of uniformly arranging control points at the edge of the survey area and arranging the control points suitable for aerial survey within the survey area can effectively reconstruct the three-dimensional reconstruction. Precision is controlled (Liu et al., 2014).

The above analysis results were carried out under the premise of aerial survey in areas with relatively flat terrain and small fluctuations. However, the archaeological site and the site area are often in the mountainous areas with complex environment. In order to meet the aerial photogrammetric control measurement specifications and even meet the higher precision standards, it is necessary to carry out the control point layout 
work according to the local conditions of the aerial survey area (Jiang, 2015).

\subsection{The impact of hardware and software equipment}

2.4.1 Selection of modeling software: Through the summarization and analysis of many 3D reconstruction software, Context Capture software is even better in 3D reconstruction. Context Capture can generate the most realistic 3D scene model from simple continuous effects without human intervention (Hua, 2019). Context Capture can calculate the ultra-high density point cloud based on the real image, and generate a high-resolution real-life three-dimensional model based on the real image bit texture. Context Capture can output common compatible formats including obj, osg (osgb), dae, etc. It can be easily imported into various mainstream GIS application platforms, and it can generate model accuracy levels of more than 20 pyramid levels. More model levels can support different cultural security efforts while performing heritage conservation and perpetual retention

\subsubsection{Hardware processing capability : The image acquired by} the drone tilt photography will be different in modeling accuracy after being processed by a computer with different hardware. (Xu, 2018). After improving the hardware performance of the processor (CPU), memory capacity, graphics card, etc., it can be seen from the experimental results that the modeling accuracy is improved after improving the processing performance of the machine, but the high-performance computer in processing time and modeling texture details Obviously better than low performance computers.

In order to better carry out the 3D reconstruction of low-altitude photogrammetry, using computer processing with better performance will reduce the model processing time and optimize the model texture detail processing to meet the working requirements.

\subsection{Summary of main factors}

Compared with urban 3D reconstruction, the site has higher requirements for the accuracy of the $3 \mathrm{D}$ reconstruction model. It is necessary to find the most critical influencing factors, and then solve and improve them to meet the requirements of the UAV mapping project. Through the collation and analysis of the influencing factors, the main influencing factors have been clearly understood and judged.

By comparison, among the many influencing factors, the most important is the correction of ground resolution and camera distortion. The higher the ground resolution of the photo, the more accurate the results will be in the third and subsequent reconstruction calculations. The setting of the ground resolution affects many other parameter settings in tilt photography, and its importance is obvious. Secondly, the distortion of the camera will also seriously affect the accuracy of the space, which directly affects the subsequent modeling accuracy. In order to obtain higher reconstruction accuracy, the camera distortion must be improved or solved.

In addition to the two factors of ground resolution and camera distortion, the other important factors are the altitude of the drone, the focal length of the camera, the size of the CCD pixel, and the layout of the image control points. The factors such as hardware and software of post-processing data will have an impact on the modeling results, but the impact is small. Therefore, the influencing factors can be ranked according to the degree of influence as shown in the following table:

\begin{tabular}{|c|l|}
\hline $\begin{array}{c}\text { Importance } \\
\text { degree }\end{array}$ & \multicolumn{1}{c|}{ influence factor } \\
\hline$\star \star \star$ & Ground Resolution , Camera Distortion \\
\hline$\star \star$ & $\begin{array}{l}\text { Flight Height, Camera Focal Length, } \\
\text { Camera CCD Pixel Size and Image } \\
\text { Control Point Layout }\end{array}$ \\
\hline$\star$ & $\begin{array}{l}\text { Unmanned Aerial Vehicle Platform, } \\
\text { Photo Overlapping, Camera Inclination, } \\
\text { Modeling Software Selection, Computer } \\
\text { Hardware Equipment }\end{array}$ \\
\hline
\end{tabular}

Table 2. Importance of Influencing Factors

In the actual three-dimensional reconstruction of tilting photography of rotorcraft, especially for heritage protection and archaeological work with higher requirements on model accuracy, it is more necessary to develop a suitable aerial survey plan according to the importance of the influencing factors shown in the table. The results obtained meet industry normative standards and meet project-related requirements. 


\section{CASE STUDY OF THREE-DIMENSIONAL RECONSTRUCTION OF SMALL AREA SITES}

The case ruins are located in the Yanqing District of Beijing. It was an ancient temple built in the Ming and Qing Dynasties. It collapsed due to years of disrepair and was naturally buried. The particularity of this temple is that it is built on another ruins. The original site was an ancient barracks and a beacon tower. It was built on a hill and surrounded by earth and stone walls to defend the enemy.

The archaeological foundations and steps of the ancient buildings were found in the archaeological site, which means that each house is at a different height during the construction. Through measurement, it was found that the height between the highest point and the lowest point of the site was nearly 10 meters. In the archaeological aerial survey of the site, the resulting photos will have a large depth of field, which may affect the accuracy of the modeling, but the sites with high differences also provide conditions for the verification experiment.

The main work of drone aerial survey in this archaeological site:

1. Three-dimensional reconstruction and digital retention of the sites and their surrounding areas during various periods of archaeological work

2. Provide archaeological mapping workers with a clear and accurate map of the orthographic image of the site.

\subsection{Digital reconstruction of the ruins area}

In order to obtain a large range of data from the site and the surrounding area, the DJI M600 multi-rotor drone is used. The drone is equipped with a five-lens measuring camera, which is specially used for the three-dimensional reconstruction of tilt photography. The UAV is limited by the flight altitude. The flight altitude is 100 meters, the heading overlap is set to $80 \%$, and the side overlap is $80 \%$. The DJI GO 4 flight control software is used to plan the route. The measurement area is approximately $120 \mathrm{~m} * 120 \mathrm{~m}$. The aerial survey data acquisition obtained a total of 1800 images, and obtained the coordinates of 9 control points for control. The Context Capture software was used for internal processing of data. The obtained model and orthophoto image are shown below

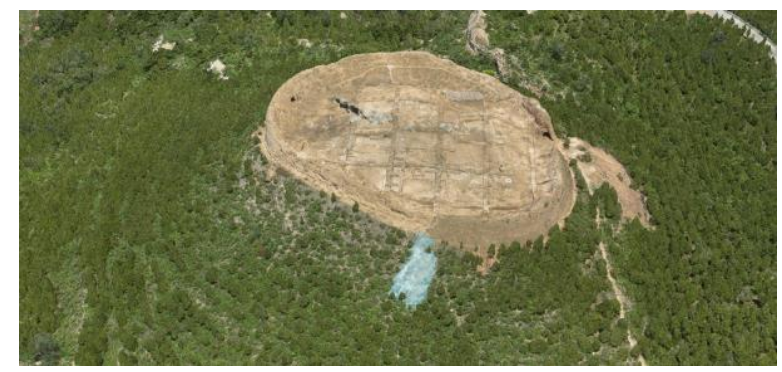

Figure 5. Site area and surrounding environment model

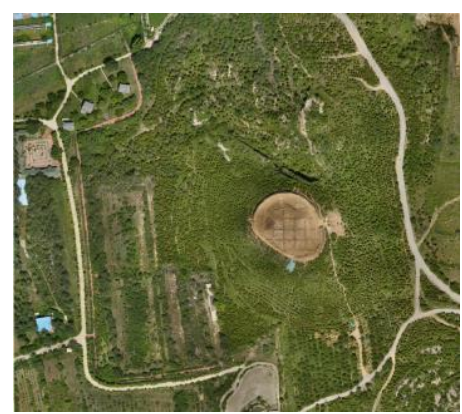

Figure 5. Orthographic image of the ruins area and surrounding environment

\subsection{Influence of height difference of survey area on reconstruction accuracy}

The archaeological area is located on the top of the mountain, and its range is about $60 \mathrm{~m} \times 60 \mathrm{~m}$. The highest difference between the highest point and the lowest point in the area is about $10 \mathrm{~m}$, which leads to the difference in the accuracy of the modeling results in the modeling area. In order to prove that the accuracy in the measurement area does vary, a simple experiment was designed. Marking paper covered with black squares of different sizes is attached to a box of fixed size as an experimental equipment. The sides of the black square are $5 \mathrm{~mm}, 10 \mathrm{~mm}$, $20 \mathrm{~mm}, 30 \mathrm{~mm}, 40 \mathrm{~mm}$ and $50 \mathrm{~mm}$ in order from small to large. Place the box at the highest and lowest points in the survey area and at other locations in the middle height. The approximate range of accuracy error in the measurement area can be judged by observing whether the black square of the identification paper after the three-dimensional reconstruction exists and is clear. The comparison of the two high and low logo layouts and modeling effects is shown in the figure below. It can be seen from the results that the height difference in the measurement area does affect the overall accuracy of the three-dimensional reconstruction. 


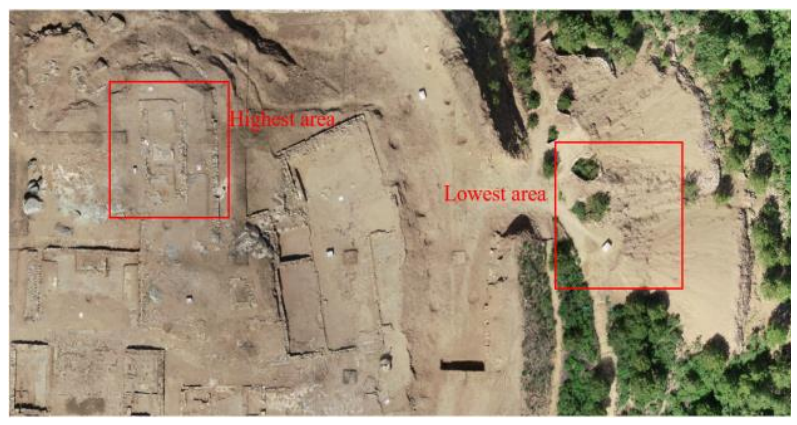

(a)

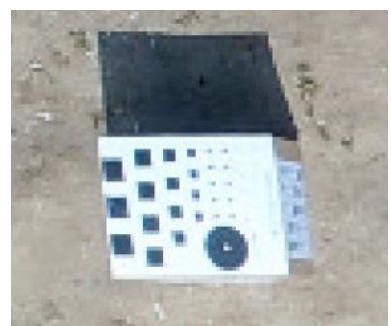

(b)

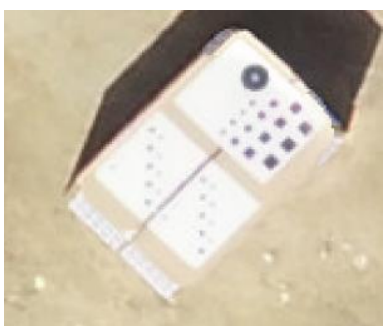

(c)
Figure 6. Comparison of the results of the highest model (b) and the lowest area (c) identification model in the site area

\subsection{HD orthophoto production in the ruins area}

In order to carry out the hand-painted archaeological maps of the ruins, the archaeological experts of the site need to cover the high-definition orthophoto images of all the ruins for reference. For the ortho-images, the gap between each tile can be clearly seen. In order to achieve higher model accuracy and orthophoto resolution, it is necessary to reduce the flying height of the drone and improve the modeling quality when data acquisition is performed.

Using the DJI Phantom 4 drone for aerial survey, the take off point is placed at the highest point of the ruins, the flight altitude is $13 \mathrm{~m}$, the heading and side overlap are $75 \%$, and 4 control points are evenly distributed around the ruins area to obtain coordinates for control. The drone carried a total of 5 routes, took a total of 90 minutes, and obtained 1683 images. The Context Capture software is also used for internal processing of data to generate an orthophoto map.

However, the orthophoto images obtained are not enough to meet the needs of archaeologists, and the details of many sites cannot be clearly expressed. However, the set flight altitude is close to the minimum requirements for flight safety, and adjusting the route of the drone alone cannot improve the accuracy of the results. In order to solve this problem, close- range photogrammetry is combined with oblique photography. The high-resolution image of the site detail obtained with the Nikon D810 camera was combined with the tilted photographic image of the drone for $3 \mathrm{D}$ reconstruction. The comparison of the orthophoto images generated before and after the close-range photogrammetry is shown in the figure below.

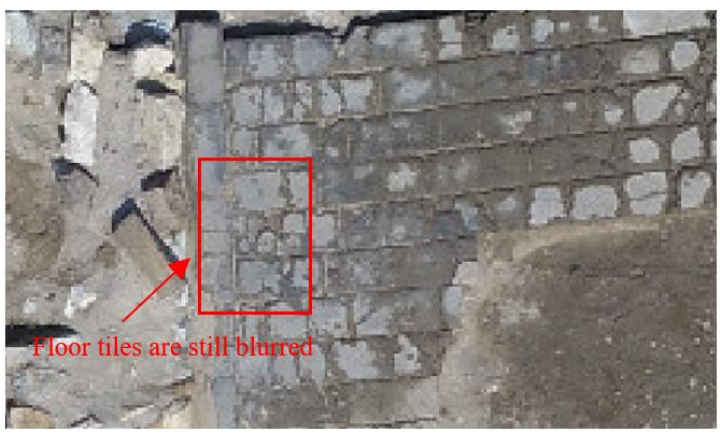

(a)

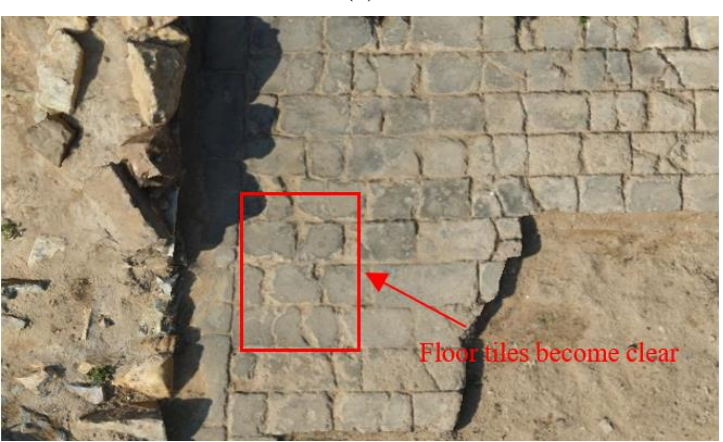

(b)

Figure 7. Three-dimensional reconstruction results before(a) and after(b) close-range photogrammetry

Through the actual three-dimensional reconstruction work, it can be proved that solving the problem of relevant influencing factors can really improve the accuracy of the three-dimensional reconstruction of the drone. However, in some cases, relying solely on the UAV tilt photography method is not able to complete the archaeological work, so it is necessary to combine other mapping methods to achieve better $3 \mathrm{D}$ reconstruction.

\section{CONCLUSIONS}

As a common wealth of all mankind, cultural heritage requires the unremitting efforts of all mankind to protect it. In SDGs 11.4, "Strengthen efforts to protect and safeguard the world's cultural and natural heritage." is also written. With the continuous advancement of society, the importance of cultural heritage protection will be recognized by more people. With the continuous development of science and technology, the 
protection of cultural heritage will become more and more efficient and more scientific.

\section{REFERENCES}

Li, S. Y., 2018. A New Exploration of Aerial Photogrammetry in Large Archaeological Sites with Small and Low-cost Unmanned Aircrafts__ Taking the Liaozhongjing Site in Chifeng as an Example. Research on Heritages and Preservation 3,128-136.

Sun, Z., Cao, Y. K., Zhang, Y. Y., 2018. Applications of Imagebased Modeling in Architectural Heritage Surveying. Research on Heritages and Preservation 3,34-40.

Sun, Z., Cao, Y. K., 2017,Accuracy Evaluation of Architectural Heritage Surveying from Photogrammetry Based on ConsumerLevel UAV_ Born Images: Case Study of the Auspicious Multi-door Stupa. Heritage Architecture 4,120-127.

Li, L. H., 2019, Large-scale 3D modeling based on drone technology_ Case Study of the Yun Gang Grottoes Scenic Area. Survey World 1,16-18.

Wu, B. T., Zhang, Y., Li, L. X., Wei, S. Q., Tian, Y., 2016. Key Technology of Oblique Images Acquisition Using Single-Lens Multicopter. Journal of Yangtze River Scientific Research Institute 33,99-103+115.

Liu, Y., 2016. Unmanned Aerial Vehicle(UAV) Oblique Photogrammetric Image Processing and 3D Modeling. East China University of Science and Technology.

Yang, W. H., Huang, B., 2018. Coping Strategies of Aerial Photogrammetry under Five-lens Tilt Condition. Site Investigation Science and Technology 3, 15-18+27.

Zhang, C. B., Yang, S. T., Zhao, C. S., 2018. Small consumer grade drone terrain data accuracy verification. Journal of Remote Sensing 22,189-199

Liao, Z. H., Peng, J. B., 2018, Discussion on Rapid Processing of Camera Distortion Parameters in Low Altitude Aerial Photography of UAV. Engineering and Technological Research 10.193-194.
Zheng, Q. H., 2015. Survey on Geometric Computing in 3D Printing. East China University of Science and Technology,1520.

Yang, Y., 2009. Research method of non-survey digital camera calibration. Science of Surveying and Mapping, 89-91.

Liao, Y. S., Chen, W. S., 2011. The Parameters Calculating and Environment Simulating System of UAV Low-Altitude Photogrammetry Operating. Bulletin of Surveying and Mapping 9, 38-41.

Jin, Y. Y., Qu, Y. C., Zhang, K. P., 2016. Three-Dimensional Modeling Accuracy Analysis Based on Overlapping Settings of Oblique Photography. Surveying and Mapping 39, 199-201+208.

Mai, X. Z., Yang, B., Feng, X. M., 2012. Discussion on the Method of Arranging Control Points for UAV Aerial Camera. Bulletin of Surveying and Mapping, 268-271.

Liu, Y. F., Zhang, X. P., Guo, Q. Y., Zhuo, X. M., Mi, Y. H., 2014. Producing Method of the Three-Dimensional Model Based on Street Factory. Geomatics \& Spatial Information Technology 12, 67-70.

Jiang, C. H., 2015. Experimental Research on Plan of Setting out Control Point for A3 Digital Camera in the Condition of Complex Topography. Bulletin of Surveying and Mapping 5, 84-86.

Hua, W., 2019. Research on Multi-resolution Color 3D Model Fusion Method. Beijing University of Civil Engineering and Architecture, 12-16.

Xu, Z. H., 2018. Feasibility Study on Three-dimensional Modeling of Tilting Photography for Consumer Unmanned Aerial Vehicles. Beijing Surveying and Mapping 32, 21-28. 